Quantum Topol. 2(2011), 173-182

DOI $10.4171 / \mathrm{QT} / 18$

Quantum Topology

(c) European Mathematical Society

\title{
A quaternionic braid representation (after Goldschmidt and Jones)
}

\author{
Eric C. Rowell ${ }^{1}$
}

\begin{abstract}
We show that the braid group representations associated with the $(3,6)$-quotients of the Hecke algebras factor over a finite group. This was known to experts going back to the 1980 s, but a proof has never appeared in print. Our proof uses an unpublished quaternionic representation of the braid group due to Goldschmidt and Jones. Possible topological and categorical generalizations are discussed.
\end{abstract}

Mathematics Subject Classification (2010). Primary 20F36; Secondary 20C08, 57M25.

Keywords. Braid group, Hecke algebra, modular category.

\section{Introduction}

Jones analyzed the images of the braid group representations obtained from Temperley-Lieb algebras in [11] where, in particular, he determined when the braid group images are finite or not. Braid group representations with finite image were also recognized in [12] and [8]. Some 15 years later the problem of determining the closure of the image of braid group representations associated with Hecke algebras played a critical role in analyzing the computational power of the topological model for quantum computation [6]. Following these developments the author and collaborators analyzed braid group representations associated with BMW-algebras [15] and twisted doubles of finite groups [5]. Partially motivated by empirical evidence the author conjectured that the braid group representations associated with an object $X$ in a braided fusion category $C$ has finite image if, and only if, the Frobenius-Perron dimension of $\mathcal{C}$ is integral (see e.g. Conjecture 6.6 of [22]). In [18], [25] various instances of this conjecture were verified. This current work verifies this conjecture

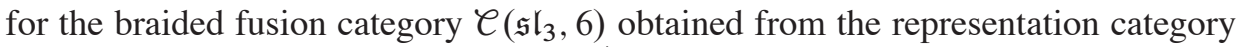
of the quantum group $U_{q} \mathfrak{s l}_{3}$ at $q=e^{\pi \mathrm{i} / 6}$ (see [23] for details and notation). More

\footnotetext{
${ }^{1}$ The author was partially supported by NSA grant H98230-10-1-0215 and benefitted from discussions with V. Jones, M. Larsen, V. Ostrik, D. Nikshych, F. Sottile and H. Wenzl. Handwritten notes by D. Goldschmidt and V. Jones were very useful.
} 
generally, Jimbo's [10] quantum Schur-Weyl duality establishes a relationship between the modular categories $\mathcal{C}\left(\mathfrak{s l}_{k}, \ell\right)$ obtained from the quantum group $U_{q} \mathfrak{s l}_{k}$ at $q=e^{\pi \mathrm{i} / \ell}$ and certain semisimple quotients $\mathscr{H}_{n}(k, \ell)$ of specialized Hecke algebras $\mathscr{H}_{n}(q)$ (defined below). That is, if we denote by $X \in \mathcal{C}\left(\mathfrak{s l}_{k}, \ell\right)$ the simple object analogous to the vector representation of $\mathfrak{s l}_{k}$ then there is an isomorphism $\mathscr{H}_{n}(k, \ell) \cong \operatorname{End}\left(X^{\otimes n}\right)$ induced by $g_{i} \rightarrow I_{X}^{\otimes i-1} \otimes c_{X, X} \otimes I_{X}^{\otimes n-i-1}$. In particular, the braid group representations associated with the modular category $\mathcal{C}\left(\mathfrak{s l}_{3}, 6\right)$ are the same as those obtained from $\mathscr{H}_{n}(3,6)$. It is known that braid group representations obtained from $\mathscr{H}_{n}(3,6)$ have finite image (mentioned in [6], [13], [18]), but a proof has never appeared in print. This fact was discovered by Goldschmidt and Jones during the writing of [8] and independently by Larsen during the writing of [6]. We benefitted from the notes of Goldschmidt and Jones containing the description of the quaternionic braid representation below. Our techniques follow closely those of [11], [12], [14]. The rest of the paper is organized into three sections. In Section 2 we recall some notation and facts about Hecke algebras and their quotients. The main results are in Section 3, and in Section 4 we indicate how the category $\mathcal{C}\left(\mathfrak{s l}_{3}, 6\right)$ is exceptional from topological and categorical points of view.

\section{Hecke algebras}

We extract the necessary definitions and results from [27] that we will need in the sequel.

Definition 2.1. The Hecke algebra $\mathscr{H}_{n}(q)$ for $q \in \mathbb{C}$ is the $\mathbb{C}$-algebra with generators $g_{1}, \ldots, g_{n-1}$ satisfying relations

(H1) $g_{i} g_{i+1} g_{i}=g_{i+1} g_{i} g_{i+1}$ for $1 \leq i \leq n-2$,

(H2) $g_{i} g_{j}=g_{j} g_{i}$ for $|i-j|>1$, and

(H3) $\left(g_{i}+1\right)\left(g_{i}-q\right)=0$.

Technically, $\mathscr{H}_{n}(q)$ is the Hecke algebra of type $A$, but we will not be considering other types so we suppress this distinction. One immediately observes that $\mathscr{H}_{n}(q)$ is the quotient of the braid group algebra $\mathbb{C} \mathscr{B}_{n}$ by the relation $(\mathrm{H} 1)^{\prime} . \mathscr{H}_{n}(q)$ may also be described in terms of the generators $e_{i}=\frac{\left(q-g_{i}\right)}{(1+q)}$, which satisfy

(H1) $e_{i}^{2}=e_{i}$,

(H2) $e_{i} e_{j}=e_{j} e_{i}$ for $|i-j|>1$, and

(H3) $e_{i} e_{i+1} e_{i}-q /(1+q)^{2} e_{i}=e_{i+1} e_{i} e_{i+1}-q /(1+q)^{2} e_{i+1}$ for $1 \leq i \leq n-2$.

For any $\eta \in \mathbb{C}$, Ocneanu [7] showed that one may uniquely define a linear functional tr on $\mathscr{H}_{\infty}(q)=\bigcup_{n=1}^{\infty} \mathscr{H}_{n}(q)$ satisfying

(1) $\operatorname{tr}(1)=1$, 
(2) $\operatorname{tr}(a b)=\operatorname{tr}(b a)$, and

(3) $\operatorname{tr}\left(x e_{n}\right)=\eta \operatorname{tr}(x)$ for any $x \in \mathscr{H}_{n}(q)$.

Any linear function on $\mathscr{H}_{\infty}$ satisfying these conditions is called a Markov trace and is determined by the value $\eta=\operatorname{tr}\left(e_{1}\right)$. Now suppose that $q=e^{2 \pi \mathrm{i} / \ell}$ and $\eta=\frac{\left(1-q^{1-k}\right)}{(1+q)\left(1-q^{k}\right)}$ for some integers $k<\ell$. Then, for each $n$, the (semisimple) quotient of $\mathscr{H}_{n}(q)$ by the annihilator of the restriction of the trace $\mathscr{H}_{n}(q) /$ Ann $(\operatorname{tr})$ is called the $(k, \ell)$-quotient. We will denote this quotient by $\mathscr{H}_{n}(k, \ell)$ for convenience. Wenzl [27] has shown that $\mathscr{H}_{n}(k, \ell)$ is semisimple and described the irreducible representations $\rho_{\lambda}^{(k, \ell)}$ where $\lambda$ is a $(k, \ell)$-admissible Young diagrams of size $n$. Here a Young diagram $\lambda$ is $(k, \ell)$-admissible if $\lambda$ has at most $k$ rows and $\lambda_{1}-\lambda_{k} \leq \ell-k$ where $\lambda_{i}$ denotes the number of boxes in the $i$ th row of $\lambda$. The (faithful) JonesWenzl representation is the sum $\rho^{(k, \ell)}=\bigoplus_{\lambda} \rho_{\lambda}^{(k, \ell)}$. Wenzl [27] has shown that $\rho^{(k, \ell)}$ is a $C^{*}$-representation, i.e. the representation space is a Hilbert space (with respect to a Hermitian form induced by the trace tr) and $\rho_{\lambda}^{(k, \ell)}\left(e_{i}\right)$ is a self-adjoint operator. One important consequence is that each $\rho_{\lambda}^{(k, \ell)}$ induces an irreducible unitary representation of the braid group $\mathscr{B}_{n}$ via composition with $\sigma_{i} \rightarrow g_{i}$, which is also called the Jones-Wenzl representation of $\mathscr{B}_{n}$.

\section{A quaternionic representation}

Consider the $(3,6)$-quotient $\mathscr{H}_{n}(3,6)$. The $(3,6)$-admissible Young diagrams have at most 3 rows and $\lambda_{1}-\lambda_{3} \leq 3$. For $n \geq 3$ there are either 3 or 4 Young diagrams of size $n$ that are $(3,6)$-admissible, and $\eta=\frac{\left(1-q^{1-3}\right)}{(1+q)\left(1-q^{3}\right)}=1 / 2$ in this case. Denote by $\varphi_{n}$ the unitary Jones-Wenzl representation of $\mathcal{B}_{n}$ induced by $\rho^{(3,6)}$. Our main goal is to prove the following:

Theorem 3.1. The image $\varphi_{n}\left(\mathscr{B}_{n}\right)$ is a finite group.

We will prove this theorem by embedding $\mathscr{H}_{n}(3,6)$ into a finite dimensional algebra (Lemma 3.2) and then showing that the group generated by the images of $g_{1}, \ldots, g_{n-1}$ is finite (Lemma 3.3). Denote by [ , ] the multiplicative group commutator and let $q=e^{2 \pi \mathrm{i} / 6}$. Consider the $\mathbb{C}$-algebra $Q_{n}$ with generators $u_{1}, v_{1}, \ldots, u_{n-1}, v_{n-1}$ subject to the relations

(G1) $u_{i}^{2}=v_{i}^{2}=-1$,

(G2) $\left[u_{i}, v_{j}\right]=-1$ if $|i-j| \leq 1$,

(G3) $\left[u_{i}, v_{j}\right]=1$ if $|i-j| \geq 2$, and

(G4) $\left[u_{i}, u_{j}\right]=\left[v_{i}, v_{j}\right]=1$. 
Notice that the group $\left\{ \pm 1, \pm u_{i}, \pm v_{i}, \pm u_{i} v_{i}\right\}$ is isomorphic to the group of quaternions. We see from these relations that $\operatorname{dim}\left(Q_{n}\right)=2^{2 n-2}$ since each word in the $u_{i}, v_{i}$ has a unique normal form

$$
\pm u_{1}^{\epsilon_{1}} \ldots u_{n-1}^{\epsilon_{n-1}} v_{1}^{v_{1}} \ldots v_{n-1}^{v_{n-1}}
$$

with $v_{i}, \epsilon_{i} \in\{0,1\}$. Observe that a basis for $Q_{n}$ is given by taking all + signs in (1). We define a $\mathbb{C}$-valued trace $\operatorname{Tr}$ on $Q_{n}$ by setting $\operatorname{Tr}(1)=1$ and $\operatorname{Tr}(w)=0$ for any non-identity word in the $u_{i}, v_{i}$. One deduces that $\mathrm{Tr}$ is faithful from the uniqueness of the normal form (1). Define

$$
s_{i}=\frac{-1}{2 q}\left(1+u_{i}+v_{i}+u_{i} v_{i}\right),
$$

for $1 \leq i \leq n-1$.

Lemma 3.2. The subalgebra $\mathcal{A}_{n} \subset Q_{n}$ generated by $s_{1}, \ldots, s_{n-1}$ is isomorphic to $\mathscr{H}_{n}(3,6)$.

Proof. It is a straightforward computation to see that the $s_{i}$ satisfy

(B1) $s_{i} s_{i+1} s_{i}=s_{i+1} s_{i} s_{i+1}$,

(B2) $s_{j} s_{i}=s_{i} s_{j}$ if $|i-j| \geq 2$, and

(E1) $\left(s_{i}-q\right)\left(s_{i}+1\right)=0$.

Indeed, relation (B2) is immediate from relations (G3) and (G4). It is enough to check (B1) and (E1) for $i=1$. For this we compute

$$
\begin{aligned}
& s_{1}^{-1}=-\frac{q}{2}\left(1-u_{1}-v_{1}-u_{1} v_{1}\right), \\
& s_{1}^{-1} u_{1} s_{1}=u_{1} v_{1}, \quad s_{1}^{-1} v_{1} s_{1}=u_{1}, \\
& s_{1}^{-1} u_{2} s_{1}=u_{2} v_{1}, \quad s_{1}^{-1} v_{2} s_{1}=-u_{1} v_{1} v_{2},
\end{aligned}
$$

from which (B1) and (E1) are deduced. Thus $\varphi\left(g_{i}\right)=s_{i}$ induces an algebra homomorphism $\varphi: \mathscr{H}_{n}(q) \rightarrow Q_{n}$ with $\varphi\left(\mathscr{H}_{n}(q)\right)=\mathcal{A}_{n}$. Set $f_{i}=\varphi\left(e_{i}\right)=\frac{\left(q-s_{i}\right)}{(1+q)}$ and let $b \in Q_{n-1}$, that is $b$ is in the span of the words in $\left\{u_{1}, v_{1}, \ldots, u_{n-2}, v_{n-2}\right\}$. The constant term of $f_{n-1} b$ is the product of the constant terms of $b$ and $f_{n-1}$ since $f_{n-1}$ is in the span of $\left\{1, u_{n-1}, v_{n-1}, u_{n-1} v_{n-1}\right\}$, so $\operatorname{Tr}\left(f_{n-1} b\right)=\operatorname{Tr}\left(f_{n-1}\right) \operatorname{Tr}(b)$. For each $a \in \mathscr{H}_{n}(q)$ we define $\varphi^{-1}(\operatorname{Tr})(a)=\operatorname{Tr}(\varphi(a))$, and conclude that $\varphi^{-1}(\operatorname{Tr})$ is a Markov trace on $\mathscr{H}_{n}(q)$. Computing, we see that $\operatorname{Tr}\left(f_{n-1}\right)=1 / 2$, so that by uniqueness $\varphi^{-1}(\mathrm{Tr})=\operatorname{tr}$ as functionals on $\mathscr{H}_{n}(q)$. Now if $a \in \operatorname{ker}(\varphi)$ we see that $\operatorname{tr}(a c)=\operatorname{Tr}(\varphi(a c))=0$ for any $c$ so that $\operatorname{ker}(\varphi) \subset \operatorname{Ann}(\operatorname{tr})$. On the other hand, if $a \in \operatorname{Ann}(\operatorname{tr})$ we must have $\operatorname{Tr}(\varphi(a c))=\operatorname{tr}(a c)=0$ for all $c \in \mathscr{H}_{n}(q)$. If $\varphi(a) \neq 0$ then, by definition of $\operatorname{Tr}$ and $\varphi$, there exists an $a^{\dagger} \in \mathscr{H}_{n}(q)$ such that $\operatorname{Tr}\left(\varphi(a) \varphi\left(a^{\dagger}\right)\right) \neq 0$ since $\operatorname{Tr}$ is faithful. Therefore $\operatorname{Ann}(\operatorname{tr}) \subset \operatorname{ker}(\varphi)$. In particular, we see that $\varphi$ induces

$$
\mathscr{H}_{n}(3,6)=\mathscr{H}_{n}(q) / \operatorname{Ann}(\operatorname{tr}) \cong \varphi\left(\mathscr{H}_{n}(q)\right)=\mathscr{A}_{n} \subset Q_{n} .
$$


Lemma 3.3. The group $G_{n}$ generated by $s_{1}, \ldots, s_{n-1}$ is finite.

Proof. Consider the conjugation action of the $s_{i}$ on $Q_{n}$. We claim that the conjugation action of $s_{i}$ on the words in the $u_{i}, v_{i}$ is by a signed permutation. Since $s_{i}$ commutes with words in $u_{j}, v_{j}$ with $j \notin\{i-1, i, i+1\}$, by symmetry it is enough to consider the conjugation action of $s_{1}$ on the four elements $\left\{u_{1}, v_{1}, u_{2}, v_{2}\right\}$, which is given in (3). Thus we see that $G_{n}$ modulo the kernel of this action is a (finite) signed permutation group. The kernel of this conjugation action lies in the center $Z\left(Q_{n}\right)$ of $Q_{n}$. Using the normal form above we find that the center $Z\left(Q_{n}\right)$ is either 1-dimensional or 4-dimensional. Indeed, since the words

$$
W=\left\{u_{1}^{\epsilon_{1}} \ldots u_{n-1}^{\epsilon_{n-1}} v_{1}^{v_{1}} \ldots v_{n-1}^{v_{n-1}}\right\}
$$

for $\left(\epsilon_{1}, \ldots, \epsilon_{n-1}, v_{1}, \ldots, v_{n-1}\right) \in \mathbb{Z}_{2}^{2 n-2}$ form a basis for $Q_{n}$ and $t w= \pm w t$ for $w, t \in W$ we may explicitly compute a basis for the center as those words $w \in W$ that commute with $u_{i}$ and $v_{i}$ for all $i$. This yields two systems of linear equations over $\mathbb{Z}_{2}$ :

$$
\left\{\begin{array}{l}
\epsilon_{1}+\epsilon_{2}=0 \\
\epsilon_{i}+\epsilon_{i+1}+\epsilon_{i+2}=0, \quad 1 \leq i \leq n-3 \\
\epsilon_{n-2}+\epsilon_{n-1}=0
\end{array}\right.
$$

and

$$
\left\{\begin{array}{l}
v_{1}+v_{2}=0, \\
v_{i-1}+v_{i}+v_{i+1}=0, \quad 1 \leq i \leq n-3, \\
v_{n-2}+v_{n-1}=0 .
\end{array}\right.
$$

Non-trivial solutions to (4) only exist if $3 \mid n$ since we must have $\epsilon_{1}=\epsilon_{2}=\epsilon_{n-2}=$ $\epsilon_{n-1}=1$ as well as $\epsilon_{i}=0$ if $3 \mid i$ and $\epsilon_{j}=1$ if $3 \nmid j$ and similarly for (5). Thus $Z\left(Q_{n}\right)$ is $\mathbb{C}$ if $3 \nmid n$ and is spanned by $1, U, V$ and $U V$ where $U=\prod_{3 \nmid i} u_{i}$ and $V=\prod_{3 \nmid i} v_{i}$ if $3 \mid n$. The determinant of the image of $s_{i}$ under any representation is a 6 th root of unity and hence the same is true for any element $z \in Z\left(Q_{n}\right) \cap G_{n}$. Thus for $3 \nmid n$ the image of any $z \in Z\left(Q_{n}\right) \cap G_{n}$ under the left regular representation is a root of unity times the identity matrix, and thus has finite order. Similarly, if $3 \mid n$, the restriction of any $z \in Z\left(Q_{n}\right) \cap G_{n}$ to any of the four simple components of the left regular representation is a root of unity times the identity matrix and so has finite order. So the group $G_{n}$ itself is finite.

This completes the proof of Theorem 3.1.

Remark 3.4. The proof of Lemma 3.3 shows that the projective image of $G_{n}$ is a (nonabelian) subgroup of the full monomial group $G\left(2,1,4^{n-1}\right)$ of signed $4^{n-1} \times 4^{n-1}$ matrices. The main goal of this paper is to verify [22], Conjecture 6.6, in this case, but with further effort one could determine the group $G_{n}$ more precisely. It is suggested 
in [13] that $G_{n}$ is an extension of $\operatorname{PSU}\left(n-1, \mathbb{F}_{2}\right)$ so that

$$
\left|G_{n}\right| \approx \frac{1}{3} 2^{(n-1)(n-2) / 2} \prod_{i=1}^{n-1}\left(2^{i}-(-1)^{i}\right),
$$

but that such a result has not appeared in print. Modulo the center, the generators $s_{i}$ have order 3 so that $G_{n} / Z\left(G_{n}\right)$ is a quotient of the factor group $\mathscr{B}_{n} /\left\langle\sigma_{1}^{3}\right\rangle$ (here $\sigma_{i}$ are the usual generators of $\left.\mathcal{B}_{n}\right)$. For $n \leq 5$, Coxeter [1] has shown that these quotients are finite groups and determined their structure. In particular, the projective image of $\mathscr{B}_{5} /\left\langle\sigma_{1}^{3}\right\rangle$ is $\operatorname{PSU}\left(4, \mathbb{F}_{2}\right)$, so $G_{5}$ is an extension of this simple group. A strategy for showing $G_{n}$ is an extension of $\operatorname{PSU}\left(n-1, \mathbb{F}_{2}\right)$ for $n>5$ would be to find an $(n-1)$-dimensional invariant subspace of $Q_{n}$ so that the restricted action of the braid generators is by order 3 pseudo-reflections (projectively). A comparison of the dimensions of the simple $\mathscr{H}_{n}(3,6)$-modules with those of $\operatorname{PSU}\left(n-1, \mathbb{F}_{2}\right)$ indicates that one must also restrict to those $n$ not divisible by 3 .

\section{Concluding remarks, questions and speculations}

The category $\mathcal{C}\left(\mathfrak{s l}_{3}, 6\right)$ does not seem to have any obvious generalizations. We discuss some of the ways in which $\mathcal{C}\left(\mathfrak{s l}_{3}, 6\right)$ appears to be exceptional by posing a number of (somewhat naïve) questions which we expect to have negative answers.

4.1. Link invariants. From any modular category one obtains (quantum) link invariants via Turaev's approach [26]. The link invariant $P_{L}^{\prime}(q, \eta)$ associated with $\mathcal{C}\left(\mathfrak{s l}_{k}, \ell\right)$ is (a variant of) the HOMFLY-PT polynomial ([7], where a different choice of variables is used). For the choices $q=e^{2 \pi \mathrm{i} / 6}$ and $\eta=1 / 2$ corresponding to $\mathcal{C}\left(\mathfrak{s l}_{3}, 6\right)$ the invariant has been identified [16]:

$$
P_{L}^{\prime}\left(e^{2 \pi \mathrm{i} / 6}, 1 / 2\right)= \pm \mathrm{i}(\sqrt{2})^{\operatorname{dim} H_{1}\left(T_{L} ; \mathbb{Z}_{2}\right)},
$$

where $T_{L}$ is the triple cyclic cover of the three sphere $S^{3}$ branched over the link $L$. There is a similar series of invariants for any odd prime $p: \pm \mathrm{i}(\sqrt{p})^{\operatorname{dim} H_{1}\left(D_{L} ; \mathbb{Z}_{p}\right)}$, where $D_{L}$ is the double cyclic cover of $S^{3}$ branched over $L$ (see [16] and [8]). It appears that this series of invariants can be obtained from modular categories $\mathcal{C}\left(\mathfrak{s o}_{p}, 2 p\right)$. This has been verified for $p=3,5$ (see [8] and [12]) and we have recently handled the $p=7$ case (unpublished, using results in [29]).

Question 4.1. Are there modular categories with associated link invariant

$$
\pm \mathrm{i}(\sqrt{p})^{\operatorname{dim} H_{1}\left(T_{L} ; \mathbb{Z}_{p}\right)} \text { ? }
$$

In [15] it is suggested that if the braid group images corresponding to some ribbon category are finite then the corresponding link invariant is classical, i.e. equivalent to a homotopy-type invariant. Another formulation of this idea is found in [24] in which classical is interpreted in terms of computational complexity. 
4.2. Fusion categories and $I_{1}$ factors. The category $C\left(\mathfrak{s l}_{3}, 6\right)$ is an integral fusion category, that is the simple objects have integral dimensions. The categories $\mathcal{C}\left(\mathfrak{s l}_{k}, \ell\right)$ are integral for $(k, \ell)=(3,6)$ and $(k, k+1)$ but no other examples are known (or believed to exist). $\mathcal{C}\left(\mathfrak{s l}_{3}, 6\right)$ has six simple (isomorphism classes of) objects: $\left\{X_{i}, X_{i}^{*}\right\}_{i=1}^{3}$ of dimension 2 (dual pairs), three simple objects $1, Z, Z^{*}$ of dimension 1 , and one simple object $Y$ of dimension 3. The Bratteli diagram for tensor powers of the generating object $X_{1}$ is given in Figure 1. It is shown in [4] that $\mathcal{C}$ is an integral fusion category if, and only if, $\mathcal{C} \cong \operatorname{Rep}(H)$ for some semisimple finite dimensional quasi-Hopf algebra $H$, so in particular $\mathcal{C}_{\left(\mathfrak{s l}_{3}, 6\right) \cong \operatorname{Rep}(H) \text { for some }}$ quasi-triangular quasi-Hopf algebra $H$. One wonders if strict coassociativity can be achieved:

Question 4.2. Is there a (quasi-triangular) semisimple finite dimensional Hopf algebra $H$ with $\mathcal{C}\left(\mathfrak{s l}_{3}, 6\right) \cong \operatorname{Rep}(H)$ ?

Other examples of integral categories are the representation categories $\operatorname{Rep}\left(D^{\omega} G\right)$ of twisted doubles of finite groups studied in [5] (here $G$ is a finite group and $\omega$ is a 3-cocycle on $G$ ). Any fusion category $C$ with the property that its Drinfeld center $\mathcal{Z}(\mathcal{C})$ is equivalent as a braided fusion category to $\operatorname{Rep}\left(D^{\omega} G\right)$ for some $\omega, G$ is called group-theoretical (see [4], [19]). The main result of [5] implies that if $\mathcal{C}$ is any braided group-theoretical fusion category then the braid group representations obtained from $\mathcal{C}$ must have finite image. In [18] we showed that $\mathcal{C}\left(\mathfrak{s l}_{3}, 6\right)$ is not group-theoretical and in fact has minimal dimension (36) among non-group-theoretical integral modular categories.

Question 4.3. Is there a family of non-group-theoretical integral modular categories that includes $\mathcal{C}\left(\mathfrak{s l}_{3}, 6\right)$ ?

Notice that $\mathcal{C}\left(\mathfrak{s l}_{3}, 6\right)$ has a ribbon subcategory $\mathscr{D}$ with simple objects $1, Z, Z^{*}$ and $Y$. The fusion rules are the same as those of $\operatorname{Rep}\left(\mathfrak{A}_{4}\right): Y \otimes Y \cong \mathbf{1} \oplus Z \oplus$ $Z^{*} \oplus Y$. However $\mathscr{D}$ is not symmetric and $\mathcal{C}\left(\mathfrak{s l}_{3}, 6\right)$ has smallest dimension among modular categories containing $\mathscr{D}$ as a ribbon subcategory (what Müger would call a minimal modular extension [17]). One possible generalization of $\mathcal{C}\left(\mathfrak{s l}_{3}, 6\right)$ would be a minimal modular extension of a non-symmetric ribbon category $\mathscr{D}_{n}$ similar to $\mathscr{D}$ above. That is, $\mathscr{D}_{n}$ should be a non-symmetric ribbon category with $n$ 1-dimensional simple objects $\mathbf{1}=Z_{0}, \ldots, Z_{n-1}$ and one simple $n$-dimensional object $Y_{n}$ such that $Y_{n} \otimes Y_{n} \cong Y_{n} \oplus \bigoplus_{i=0}^{n-1} Z_{i}$ and the $Z_{i}$ have fusion rules like $\mathbb{Z}_{n}$. For $\mathscr{D}_{n}$ to exist even at the generality of fusion categories one must have $n=p^{\alpha}-1$ for some prime $p$ and integer $\alpha$ by [3], Corollary 7.4. However, V. Ostrik [20] informs us that these categories do not admit non-symmetric braidings except for $n=2,3$. So this does not produce a generalization. A pair of hyperfinite $I I_{1}$ factors $A \subset B$ with index $[B: A]=4$ can be constructed from $\mathcal{C}\left(\mathfrak{s l}_{3}, 6\right)$ (see [28], Section 4.5). The corresponding principal graph is the Dynkin diagram $E_{6}^{(1)}$ the nodes of which we 


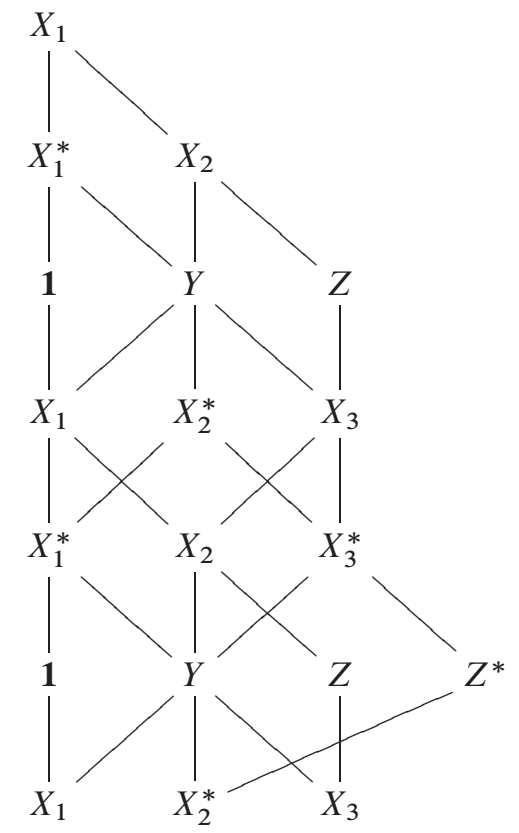

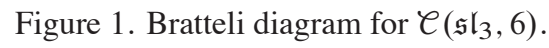

label by simple objects:

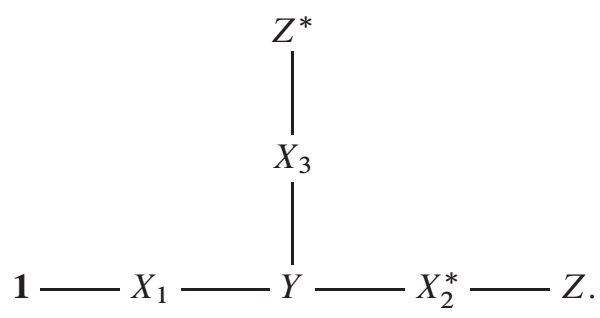

This principal graph can be obtained directly from the Bratteli diagram in Figure 1 as the nodes in the 6th and 7th levels and the edges between them. Hong [9] showed that any $I I_{1}$ subfactor pair $M \subset N$ with principal graph $E_{6}^{(1)}$ can be constructed from some $I I_{1}$ factor $P$ with an outer action of $\mathfrak{A}_{4}$ as $M=P \rtimes \mathbb{Z}_{3} \subset P \rtimes \mathfrak{A}_{4}=N$. Subfactor pairs with principal graph $E_{7}^{(1)}$ and $E_{8}^{(1)}$ can also be constructed (see e.g. [21]). We ask:

Question 4.4. Is there a unitary non-group-theoretical integral modular category with principal graph $E_{7}^{(1)}$ or $E_{8}^{(1)}$ ?

Even a braided fusion category with such a principal graph would be interesting, and have interesting braid group image. Notice that the subcategory $\mathscr{D}$ mentioned 
above plays a role here as $\mathfrak{A}_{4}$ corresponds to the Dynkin diagram $E_{6}^{(1)}$ in the McKay correspondence. A modular category $C$ with principal graph $E_{7}^{(1)}$ (resp. $E_{8}^{(1)}$ ) would contain a ribbon subcategory $\mathscr{F}_{1}\left(\operatorname{resp} . \mathcal{F}_{2}\right)$ with the same fusion rules as $\operatorname{Rep}\left(\mathfrak{S}_{4}\right)$ (resp. Rep $\left.\left(\mathfrak{A}_{5}\right)\right)$. Using [2], Lemma 1.2, we find that such a category $\ell$ must have dimension divisible by 144 (resp. 3600). The ribbon subcategory $\mathcal{F}_{2}$ must have symmetric braiding (D. Nikshych's proof: $\operatorname{Rep}\left(\mathfrak{A}_{5}\right)$ has no non-trivial fusion subcategories so if it has a non-symmetric braiding, the Müger center is trivial. But if the Müger center is trivial it is modular, which fails by [2], Lemma 1.2). This suggests that for $E_{8}^{(1)}$ the answer to Question 4.4 is "no." There is a non-symmetric choice for $\mathscr{F}_{1}$ (as V. Ostrik informs us [20]), with Müger center equivalent to $\operatorname{Rep}\left(\mathfrak{S}_{3}\right)$. By [17], Proposition 5.1, a minimal modular extension $\mathcal{C}$ of such an $\mathcal{F}_{1}$ would have dimension 144.

\section{References}

[1] H. S. M. Coxeter, Factor groups of the braid group. In Proceedings of the Fourth Can. Math. Congress, Banff 1957, University of Toronto Press, (1959), 95-122. Zbl 0093.25003

[2] P.Etingof and S. Gelaki, Some properties of finite-dimensional semisimple Hopf algebras. Math.Res. Lett. 5 (1998), 191-197. MR 1617921 Zbl 0907.16016

[3] P. Etingof, S. Gelaki, and V. Ostrik, Classification of fusion categories of dimension $p q$. Int. Math. Res. Not. (2004), 3041-3056. MR 2098028 Zbl 1063.18005

[4] P. Etingof, D. Nikshych, and V. Ostrik, On fusion categories. Ann.of Math. (2) 162 (2005), 581-642. MR 2183279 Zbl 1125.16025

[5] P. Etingof, E. Rowell, and S. Witherspoon, Braid group representations from twisted quantum doubles of finite groups. Pacific J. Math. 234 (2008), 33-41. MR 2375313 Zbl 05366298

[6] M. H. Freedman, M. J. Larsen, and Z. Wang, The two-eigenvalue problem and density of Jones representation of braid groups. Comm. Math. Phys. 228 (2002), 177-199. MR 1911253 Zbl 1045.20027

[7] P. Freyd, D. Yetter, J. Hoste, W. B. R. Lickorish, K. Millett, and A. Ocneanu, A new polynomial invariant of knots and links. Bull. Amer. Math. Soc. (N.S.) 12 (1985), 239246. MR 776477 Zbl 0572.57002

[8] D. M. Goldschmidt and V. F. R. Jones, Metaplectic link invariants. Geom. Dedicata 31 (1989), 165-191. MR 1012438 Zbl 0678.57007

[9] J. H. Hong, Subfactors with principal graph $E_{6}^{(1)}$. Acta Appl. Math. 40 (1995), 255-264. MR 1340721 Zbl 0827.46051

[10] M. Jimbo, A $q$-analogue of $U(\mathfrak{g l}(N+1))$, Hecke algebra, and the Yang-Baxter equation. Lett. Math. Phys. 11 (1986), 247-252. MR 841713 Zbl 0602.17005

[11] V. F. R. Jones, Braid groups, Hecke algebras and type $\mathrm{II}_{1}$ factors. In Geometric methods in operator algebras (Kyoto, 1983), Pitman Res. Notes Math. Ser. 123, Longman Sci. Tech., Harlow 1986, 242-273. Zbl 0659.46054 
[12] V. F. R. Jones, On a certain value of the Kauffman polynomial. Comm. Math. Phys. 125 (1989), 459-467. MR 1022523 Zbl 0695.57003

[13] M. Larsen and E. Rowell, Unitary braid representations with finite image. Algebr. Geom. Topol. 8 (2008), 2063-2079. MR 2452917 Zbl 1187.20047

[14] M. J. Larsen and E. C. Rowell, An algebra-level version of a link-polynomial identity of Lickorish. Math. Proc. Cambridge Philos. Soc. 144 (2008), 623-638. MR 2418707 Zbl 1154.57007

[15] M.J.Larsen,E.C. Rowell, and Z. Wang, The $N$-eigenvalue problem and two applications. Int. Math. Res. Not. (2005), 3987-4018. MR 2206918 Zbl 1109.22008

[16] W. B. R. Lickorish and K. C. Millett, Some evaluations of link polynomials. Comment. Math. Helv. 61 (1986), 349-359. MR 860127 Zbl 0607.57003

[17] M. Müger, On the structure of modular categories. Proc.London Math. Soc. (3) 87 (2003), 291-308. MR 1990929 Zbl 1037.18005

[18] D. Naidu and E. C. Rowell, A finiteness property for braided fusion categories. To appear in Algebr. Represent. Theory.

[19] S. Natale, On group theoretical Hopf algebras and exact factorizations of finite groups. J. Algebra 270 (2003), 199-211. MR 2016657 Zbl 1040.16027

[20] V. Ostrik, private communication.

[21] S. Popa, Sur la classification des sous-facteurs d'indice fini du facteur hyperfini. C. $R$. Acad. Sci. Paris Sér. I Math. 311 (1990), 95-100. MR 1065437 Zbl 0706.46048

[22] E. Rowell, R. Stong, and Z. Wang, On classification of modular tensor categories. Comm. Math. Phys. 292 (2009), 343-389. MR 2544735 Zbl 1186.18005

[23] E. C. Rowell, From quantum groups to unitary modular tensor categories. In Representations of algebraic groups, quantum groups, and Lie algebras, Contemp. Math. 413, Amer. Math. Soc., Providence, RI 2006, 215-230. Zbl 1156.18302

[24] E. C. Rowell, Two paradigms for topological quantum computation. In Advances in quantum computation, Contemp. Math. 482, Amer. Math. Soc., Providence, RI 2009, 165-177. Zbl 1171.81344

[25] E. C. Rowell, Braid representations from quantum groups of exceptional Lie type. Rev. Un. Mat. Argentina 51 (2010), 165-175. MR 2681262

[26] V. G. Turaev, Quantum invariants of knots and 3-manifolds. de Gruyter Studies in Mathematics 18, Walter de Gruyter \& Co., Berlin 1994. MR 1292673 Zbl 0812.57003

[27] H. Wenzl, Hecke algebras of type $A_{n}$ and subfactors. Invent. Math. 92 (1988), 349-383. MR 936086 Zbl 0663.46055

[28] H. Wenzl, $C^{*}$ tensor categories from quantum groups. J. Amer. Math. Soc. 11 (1998), 261-282. MR 1470857 Zbl 0888.46043

[29] B. W. Westbury, Invariant tensors for the spin representation of $\mathfrak{s o}$ (7). Math. Proc. Cambridge Philos. Soc. 144 (2008), 217-240. MR 2388243 Zbl 05249387

Received June 1, 2010

Eric C. Rowell, Mathematics Department, Texas A\&M University, College Station, TX 77843-3368, U.S.A.

E-mail: rowell@math.tamu.edu 